\title{
Distributed Association Method Assisted by Cell for Efficiency Enhancement of Wireless Networks
}

\author{
Jaesung Park \\ Department of Information Security, University of Suwon, San 2-2, Wau-ri, Bongdam-eup, Hwaseong-si, \\ Gyeonggi-do 445-743, Republic of Korea
}

Correspondence should be addressed to Jaesung Park; jaesungpark@suwon.ac.kr

Received 22 November 2016; Accepted 24 January 2017; Published 16 February 2017

Academic Editor: Hideyuki Takahashi

Copyright (C) 2017 Jaesung Park. This is an open access article distributed under the Creative Commons Attribution License, which permits unrestricted use, distribution, and reproduction in any medium, provided the original work is properly cited.

In this paper, we propose a distributed cell association scheme called cell-guided association method (CGAM) to improve the efficiency of a wireless network. In CGAM, MSs attempt to associate with their best cells. However, unlike the conventional methods, cells do not passively accept the association requests of MSs. Instead, a cell determines whether to accept an association request or not by considering the performance of MSs already associated with it and that of the requesting MS. If a cell cannot provide a certain level of service to them, it rejects the association request and guides the requesting MS to select another cell that gives the next maximum performance metric to the MS. Since our method takes the cell resource usage into consideration, it can increase the resource efficiency of a wireless network while enhancing the overall data rate provided to MSs by balancing the number of MSs in a cell. Through performance comparisons by simulation studies, we verify that CGAM outperforms maximum SINR-based method and QoS-based method in terms of the total data rate provided by a system and outage probabilities of MSs.

\section{Introduction}

As the number of handheld devices proliferates fast, mobile data traffic has increased tremendously over a few decades. The traffic demand is expected to grow over the future because of the advent of Internet of things [1, 2]. To cope with the traffic demands, wireless networks become more and more complex and the importance of distributed resource management methods that increase both the resource efficiency of a network and quality of service (QoS) experiences by users attracts much attention. Among those, since a cell association policy that matches a cell and a mobile station (MS) affects the distribution of MSs, it influences system efficiency and QoS of users.

Traditionally, MSs attempt to associate with a cell that give them the strongest signal-to-interference and noise ratio (SINR). The rationale behind this is that data rate provided to a MS enhances as the signal quality between a MS and a cell increases [3]. However, the data rate provided to a MS is determined not only by the SINR but also by the amount of radio resources allocated to it. A MS can measure SINRs from its adjacent cells. However, a MS does not know the amount of resources that it can obtain from a cell before it associates with the cell. Since cell resources are shared by the MSs associated with a cell, the fraction of resources allocated to each MS decreases as the number of MSs in a cell increases. Therefore, if a MS selects a cell to associate with based on the SINR, it may not receive the highest data rate. In addition, SINR between a cell and a MS is determined by the transmit power of a cell, the level of interference, and the random fading. Because of the randomness, it becomes highly probable that the number of MSs in a cell is not evenly distributed among cells even if cells are carefully deployed considering the traffic demands of areas covered by them. If the load unbalance among cells occurs, MSs in a lightly populated cell enjoy high data rate while MSs in a densely populated cell suffer from low data rate because of the resource contention among MSs.

To overcome this problem, cell load-aware or QoSoriented cell association methods have been proposed. In [4], joint optimization of user association policies and antenna tilts settings is proposed by predicting cell load accurately. The authors in [5] propose two distributed association methods that enable MSs to make association decisions based on the information gathered by probing neighboring cells. In [6], 
a use association policy is proposed to minimize the energy consumption in LTE access networks which are composed of small cells deployed densely. However, cell load-aware association methods require that cells broadcast the cell loads periodically or on-demand. In addition, MSs select a cell to associate with to maximize its benefit selfishly while cells passively accept the association request from MSs. Therefore, system resources are not used in an optimal way because of the selfishness.

To address this issue, we devise a distributed cell-guided association method (CGAM). In CGAM, cells do not passively accept the association requests from MSs. On the contrary, cells determine whether or not to accept the association request based on their resource availability. Thus, even if MSs operate selfishly to maximize its payoff, system can guide MSs to achieve its own objective such as increasing resource utilization and cell load balance. In addition, since CGAM does not require cells to broadcasts cell load, conventional cell operations need not be changed.

The rest of the paper is organized as follows. In Section 2, we review related works on association management. In Section 3, we describe the CGAM in detail after introducing system model. In Section 4, we present and discuss simulation results to verify CGAM by comparing the performances of CGAM and SINR-based association method and cell loadaware association method.

\section{Related Works}

Since the cell association problem is to find a set of MSs and cells pairs that optimize a given objective function of a wireless network, optimization methods are widely used to design an association rule. Various objective functions are devised for the cell association problem. In [7], authors propose an algorithm that maximizes the system revenue while associating MSs with the minimum total transmission power. They use Benders' decomposition to solve nonconvex optimization problem optimally. Throughput maximization is also used as an objective function. In [8], sum rate is maximized and authors in $[9,10]$ maximize the log-utility of a network under the proportional fairness. In [11], MSs are associated with BSs in a way that global outage probability is minimized. However, it has been shown that the optimization problem is an NP-hard problem.

To overcome the NP-hardness, different kinds of optimization techniques are used to solve the cell association problem. Markov decision process is used in [12, 13]. However, it is hard to define state transition probability and the complexity increases substantially as the number of states increases. In [14], the structure of the network utility maximization problem is used to solve the problem directly. Dual decomposition is often employed to design a distributed algorithm by relaxing the optimization constraints $[10,15]$. However, since the computational cost of these methods is high, it is not clear whether these methods can be applied at a small time scale when the values of input variables change very fast.

Game theory is also applied to solve the cell association problem. In [16], an evolutionary game theory is used to devise an algorithm for cell association and antenna allocation in $5 \mathrm{G}$ networks with massive MIMO. Authors in [17] show that the game that users selfishly select base stations giving them the best throughput and BSs allocate the same time to their users has one Nash equilibrium point which achieves proportional fairness system-wide. However, the convergence of the algorithms based on a game theory is not guaranteed generally. Additionally, in terms of the implementation, they usually cause large overhead, which deteriorates resource utilization.

Stochastic geometry has been used to analyze the cell association problem. In [18], authors propose a distributed belief propagation algorithm to resolve user association problem and analyze the average sparsity and degree distribution using stochastic geometry. Stochastic geometry is also used in [19] to analyze the service success probability. Then, they derive the impact of cell association and user scheduling on the service success probability. Unlike the optimization methods that maximize the utility function for the current network configuration, stochastic geometry performs optimization over the average utility. Therefore, even though the complexity and overhead of a stochastic geometry approach are lower than those of repeating optimization process whenever network configuration changes, the results will be suboptimal.

Unlike the association methods based on a theoretical static interference model, a practical measurement based interference-aware association policy is proposed in [20].

There have been research works that optimize jointly cell association and other performance metrics. In [21], a tractable framework is proposed to analyze the performance of eICIC by jointly considering cell association, resource partitioning, and transmit power reduction. An energy efficient user association scheme is proposed in [22, 23]. Joint BS association and power control algorithm that updates iteratively BS association solution then the transmit power of each user is proposed in [24].

\section{Cell-Guided Association Method}

3.1. System Model. We consider a downlink of a wireless network that provides best effort data service to MSs. We denote by $N_{c}$ the set of cells in a network and by $\left|N_{c}\right|$ the cardinality of $N_{c}$. We assume that the system fully reuses frequency.

Since radio resources of a cell are allocated to MSs in the unit of radio resource block (RB) in modern wireless networks such as LTE, we assume that each cell $x$ has $R_{x}$ RBs. We consider long term SINR and throughput. In other words, we assume that the timescale measuring these values is much larger than that of fast channel variation. Thus, if we denote the transmit power of a cell $x$ as $P_{x}$, the SINR between a MS $y$ and a cell $x$ is given by

$$
S_{x, y}=\frac{P_{x} G_{x, y}}{A_{N}+\sum_{c \in N_{c}-\{x\}} P_{c} G_{c, y}},
$$

where $A_{N}$ is the noise power and $G_{x, y}$ denotes the channel gain between a cell $x$ and a MS $y$ which includes the system 
parameter such as antenna gain and channel parameters such as path loss and shadowing.

The spectral efficiency is an increasing function of $S_{x, y}$. A variety of functions have been devised to reflect the effect of operational frequency and modulation and coding schemes [25-27]. However, to avoid system dependency, we use Shannon's formula to obtain the data rate for given SINR. If a cell $x$ allocates $R_{x, y}$ RBs to a MS $y$, the data rate of $y$ is obtained as

$$
D_{x, y}=R_{x, y} W_{R} \log _{2}\left(1+S_{x, y}\right),
$$

where $W_{R}$ is the bandwidth of a RB. The number of RBs allocated to a MS is determined by the type of scheduler a cell uses. A scheduler considers the number of MSs sharing cell resources, the channel quality between a cell, and a MS when it allocates resources at each frame. In case of a round-robin scheduler, the long term RBs allocated to each MS becomes $R_{x} /\left|M_{x}\right|$, where $M_{x}$ is the set of MSs that a cell $x$ serves.

However, it is shown that there is a multiuser diversity gain when a cell exploits a proportional fair scheduler [2830]. In this paper, we assume a cell uses a proportional fair scheduler. Then according to the results of [30], $R_{x, y}$ is determined as follows.

$$
R_{x, y}=\frac{R_{x}}{\left|M_{x}\right|} g\left(M_{x}\right),
$$

where $g\left(M_{x}\right)=\sum_{i=1}^{\left|M_{x}\right|}(1 / i)$ is the multiuser diversity gain.

3.2. QoS Metric. When a MS requests for a guaranteed service such as a voice call, the number of RBs to provide the service is guaranteed by a cell. Moreover, no more RBs are allocated to the request even if a cell has surplus RBs. However, when a cell provides data service, the number of RBs allocated to each MS varies according to the number of MSs served by a cell simultaneously. For example, even when there is only one MS associated with a cell, the cell allocates all its RBs to the MS. We also note that even if a MS uses data service, users tend to give up using a network when they did not receive a minimum rate from a network. Thus, we use the outage probability [31-33] as the QoS metric for a MS. The outage probability is defined as the probability that a data rate provided to a MS is less than a given threshold $\theta_{d}$. We use the outage probability model derived in a network whose frequency reuse factor is one and where rayleigh fading channel is assumed [33]. When a MS $y$ associates with a cell $x$, the outage probability of $y$ is modeled as

$$
\begin{aligned}
O_{x, y}= & \operatorname{Prob}\left(D_{x, y}<\theta_{d}\right) \\
= & 1 \\
& \quad-e^{\frac{A_{N} B_{x, y}}{G_{x, y} P_{x}}} \prod_{i \in N_{c}-\{x\}} \frac{1 /\left(G_{i, y} P_{i}\right)}{B_{x, y} /\left(G_{x, y} P_{x}\right)+1 /\left(G_{i, y} P_{i}\right)},
\end{aligned}
$$

where $B_{x, y}=2^{\theta_{d} / D_{x, y}}-1$. Since $O_{x, y}$ is a decreasing function of $D_{x, y}, O_{x, y}$ decreases if $R_{x, y}$ or $S_{x, y}$ increases. Therefore $O_{x, y}$ can be regarded as a metric that represents the degree of cell load.
3.3. CGAM Algorithm. CGAM is composed of two algorithms, a MS algorithm and a cell algorithm, that operate independently of each other. Algorithm 1 shows each algorithm.

A MS that needs to associate with a cell searches neighboring cells by hearing signals sent by them. Then, a MS $y$ constructs a set of adjacent cells $\left(C_{y}\right)$ that contains the cell identification numbers and SINR received from each cell in $C_{y}$. Then, $y$ initializes a potential cell list $C_{y}^{\prime}=C_{y}$ and attempts to associate with a cell $x$ in $C_{y}^{\prime}$ that gives it the maximum SINR by sending an association request message and waits for the response from $x$. If $y$ receives an association response message from $x$ saying that $x$ does not accept the request, $y$ removes the cell $x$ in $C_{y}^{\prime}$ and attempts to associate with a cell in $C_{y}^{\prime}$ giving the highest SINR to it (i.e., the second best cell in $C_{y}$ in terms of SINR). A MS $y$ repeats the same process until it receives a positive association response from a cell. If $y$ does not receive a positive response from any cell in $C_{y}$, it means that $y$ cannot be provided with the minimum data rate from its neighboring cells. Thus, it suspend the association attempt.

Upon receiving an association request message from a MS $y$, a cell $x$ calculates the outage probabilities of $y$ and $j$ in $M_{x}$ by assuming that it accepts the request. If none of the outage probabilities are below a threshold value $\theta_{o}, x$ accepts the association request of $y$ by sending a positive association response message to $y$. Otherwise, $x$ guides $y$ to associate with other cells that may satisfy the outage probability constraint by sending a negative association response to $y$.

\section{Performance Evaluation}

In this section, we evaluate the performance of the proposed cell association methods. Specifically we compare the performance of the following three methods in terms of the outage probability of a MS and data rates provided by cells.

(i) SNM: a MS associates with a cell according to the maximum signal strength and a cell does not guide a MS to associate with better cell.

(ii) ONM: a MS associates with a cell based on the minimum outage probability and a cell does not guide a MS to associate with better cell.

(iii) SOM: a MS associates with a cell based on the maximum signal strength and a cell guide a MS to associate with better cell with the outage probability it can provide to an MS.

We construct urban macrocell topology following the scenario specified in 3GPP [27]. We deployed 18 base stations. Each base station has three sectors. We assume a hexagonal cell and each cell has 6 neighboring cells. The system bandwidth is set to $5 \mathrm{MHz}$, and the bandwidth of a RB is configured as $180 \mathrm{KHz}$. The intersite distance is set to $500 \mathrm{~m}$. Transmit power of a base station is configured to be $46 \mathrm{dBm}$. Antenna gain of a base station is $45 \mathrm{dBi}$ and that of a MS is $2 \mathrm{dBi}$. The noise power is configured as $-111.45 \mathrm{dBm}$ and frequency reuse factor is set to 1 . 
MS Algorithm

(0) cell search

(1) construct $C_{y}$

(2) $C_{y}^{\prime} \leftarrow C_{y}$

(3) While $C_{y}^{\prime} \neq \varnothing$

(4) $x \leftarrow \operatorname{argmax} i \in C_{y}^{\prime}\left(S_{i, y}\right)$

(5) send association request message to $x$

(6) receive association response message from $x$

(7) if (response $\neq \mathrm{OK}$ )

(8) $\quad C_{y}^{\prime} \leftarrow C_{y}^{\prime}-\{x\}$

Cell Algorithm

(0) Upon receiving association request from a MS $y$

(1) $M_{x}^{\prime} \leftarrow M_{x} \cup\{y\}$

(2) flag $\leftarrow 0$

(3) while $M_{x}^{\prime} \neq \varnothing$

(4) calculate $O_{x, j},\left(j \in M_{x}^{\prime}\right)$

(5) if $\left(O_{x, j}>\theta_{o}\right)$

(6) send association response with NOK

(7) flag $\leftarrow 1$

(8) break

(9) else

(10) $M_{x}^{\prime} \leftarrow M_{x}-\{i\}$

(11) if (flag $==0$ )

(12) send association response with OK

Algorithm 1: Cell-guided association method.
The path loss model of $128.1+37.6 \log _{10}(\max (d, 0.035))$ is applied, where $d$ is the distance in $\mathrm{km}$ between a sender and a receiver. We assume log-normal shadowing channel that has zero mean and standard deviation of $\sigma_{\mathrm{dB}}=8 \mathrm{~dB}$.

We locate a MS in a network randomly following the uniform distribution and apply each method for a MS to determine a cell to associate with. All the statistics are gathered when the number of MSs reach 5,000.

4.1. MS Performance. To quantify the performance of MS, we use two metrics. One metric is the data rate received by a MS and the other is the outage probability of a MS. According to the locations of MSs, we further classify MSs into edge MSs which are defined as the MSs whose Euclidean distance from its serving base station is larger than intersite distance over three.

Figure 1 shows the cumulative distribution of data rates received by MSs and Figure 2 shows the cumulative distribution of outage probabilities experienced by MSs. Comparing SNM with ONM, performances of MSs increase by changing cell selection metric from SINR to outage probability. For example, 70\% MSs receive less than 575.8 Kbps when SINR is used as a cell selection metric. On the contrary $70 \% \mathrm{MSs}$ receive $607.9 \mathrm{Kbps}$ when MSs use outage probability to select a cell. In terms of the outage probability, the proportion of MSs whose outage probabilities are less than 0.2 is 0.794 when SINR is used. On the contrary, the proportion increases to 0.817 when MS selects a cell with an outage probability.

The performances of MSs increase dramatically when a cell guides MSs when they select a cell to associate with. When
TABLE 1: Cell statistics in terms of the number of MSs in a cell.

\begin{tabular}{lccc}
\hline & SNM & ONM & SOM \\
\hline FI & 0.88 & 0.96 & 0.92 \\
Avg. & 215 & 208 & 137 \\
Std. & 80 & 41 & 43 \\
\hline
\end{tabular}

the association method is changed from SNM to SOM, the data rate received by $70 \%$ MSs increases from $575.8 \mathrm{Kbps}$ to $904.2 \mathrm{Kbps}$. In addition, the proportion of MSs whose outage probabilities are less than 0.2 increases 1.06 times from 0.794 to 0.842 .

The performance gain obtained by guiding MSs with outage probability is more significant for the edge MSs. Ninety percentage of edge MSs receive less than 793.3 Kbps when SNM is used while it increases to $1301.9 \mathrm{Kbps}$ when SOM is used. The outage probabilities of $90 \%$ edge MSs are less than 0.5 when SNM is applied while the number decreases to 0.37 when MSs use SOM to associate with a cell.

4.2. System Performance. Figure 3 shows the total data rate provided by each cell called cell rate. We can observe that the cell rate increase dramatically if there is a guide by a cell. This is attributed to the fact that if MSs choose cells selfishly to maximize their own performance metric, some cells are crowded with MSs while the others are loosely populated. To examine the distribution of MSs in a cell, in Table 1, we show Jain's fairness index (FI) in terms of the number of MSs in a cell. In the table, we also show the average and the standard 


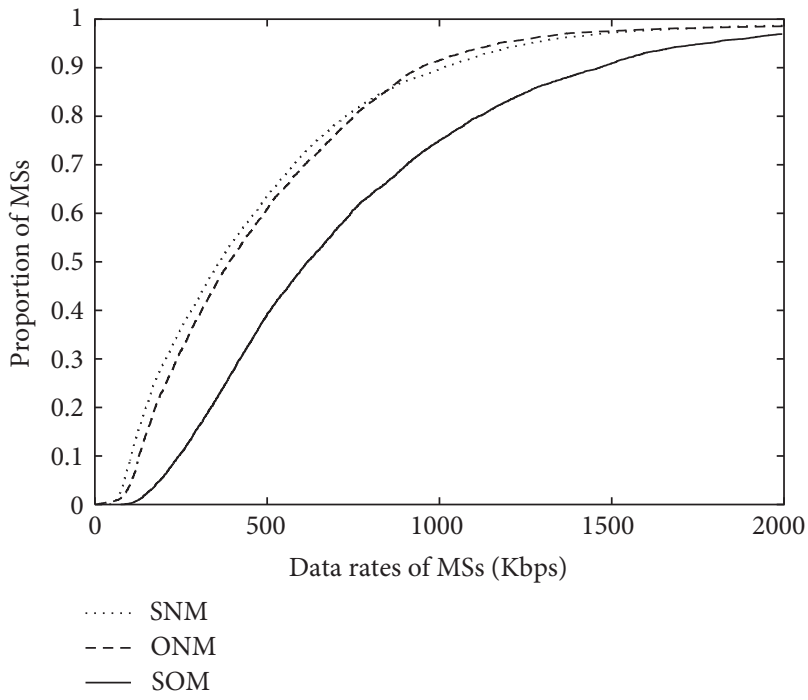

(a) All MSs

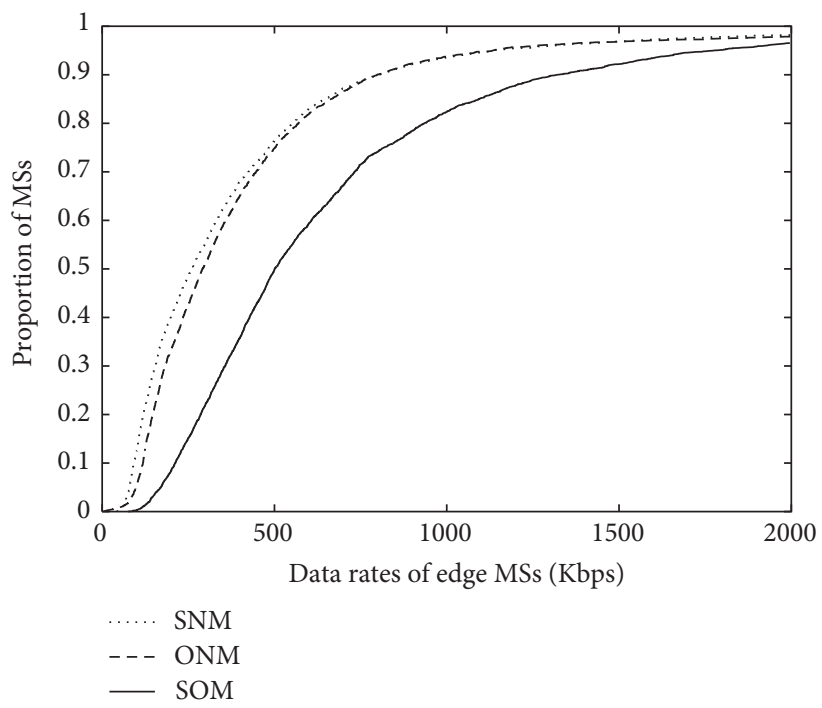

(b) Edge MSs

FIgURE 1: Cumulative distribution of data rates received by MSs.

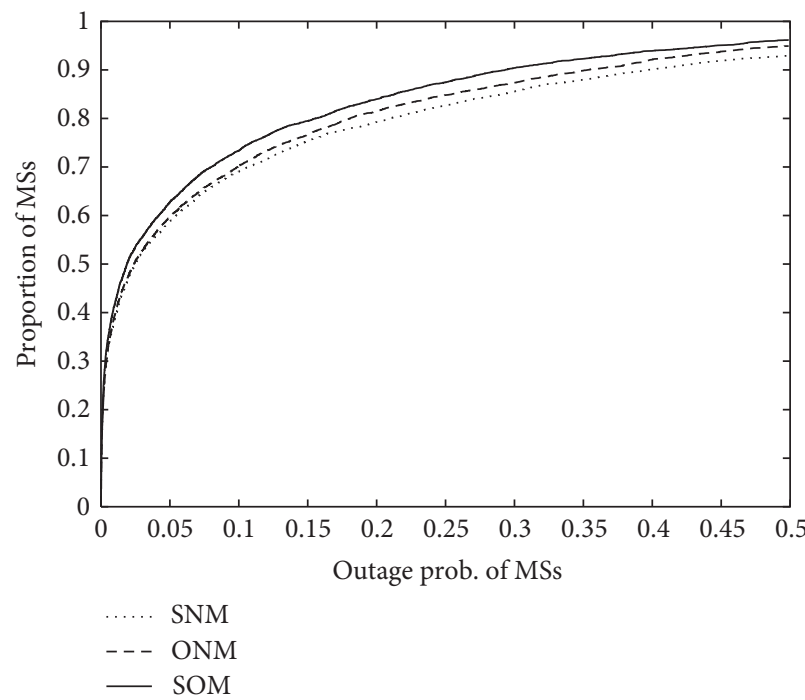

(a) All MSs

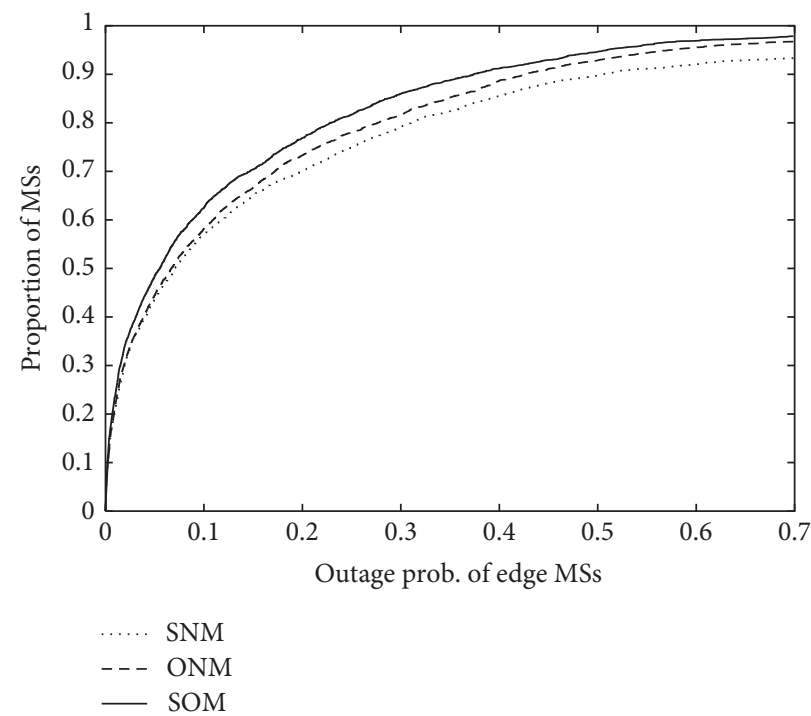

(b) Edge MSs

FIGURE 2: Cumulative distribution of MS outage probability.

deviation of the number of MSs in a cell. We can find that FI is 0.88 and the standard deviation is 80 when SNM is used while FI and the standard deviation of SOM are 0.92 and 43, respectively, which means that MSs are more evenly populated when SOM is used than when SNM is used. ONM also distribute MSs evenly. However, the average number of MSs in a cell of ONM is higher than those of SOM. Since cell resources are shared among MSs, the number of RBs that is allocated to each MS in a cell becomes smaller as the number of MSs in a cell increases. In addition, since SINR is inversely proportional to the distance from a base station and a MS and we deployed MSs according to the uniform distribution, the average data rate per RB decreases as the number of MSs in a cell increases. Therefore, even though cell rate of ONM is higher than that of SNM, it is smaller than the cell rate of SOM.

To further investigate the benefit of cell guidance, we show the minimum data rate provided to a MS by each cell in Figure 4 and the maximum outage probability among the MSs in each cell experience in Figure 5. We can observe that the minimum data rate provided to MSs in a cell increases and the maximum outage probability in each cell decreases. 


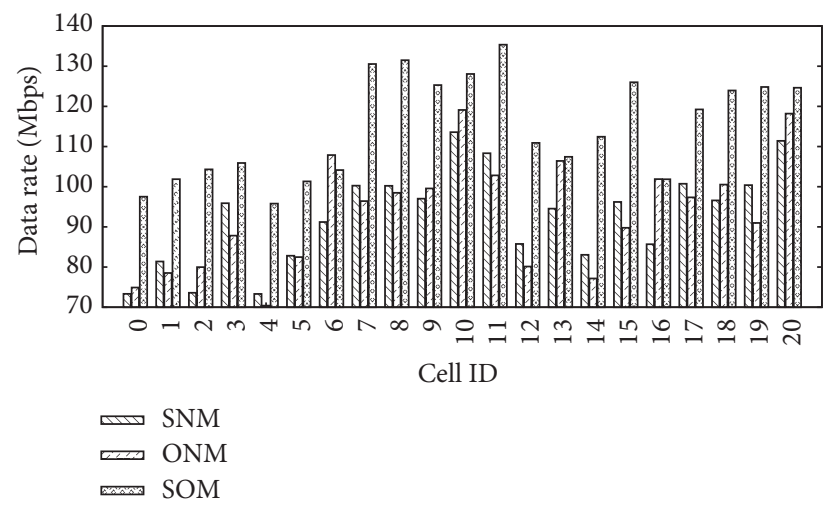

FIgURE 3: Total data rate provided by a cell.

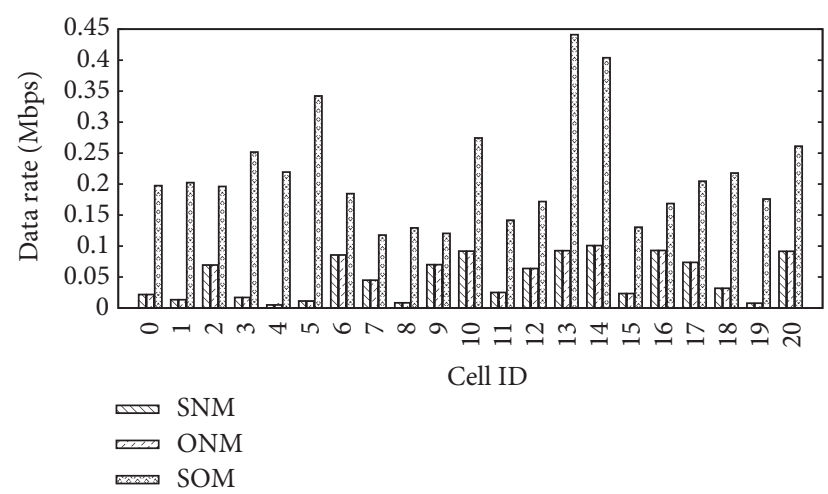

FIgURE 4: Minimum data rate provided to a MS associated with a cell.

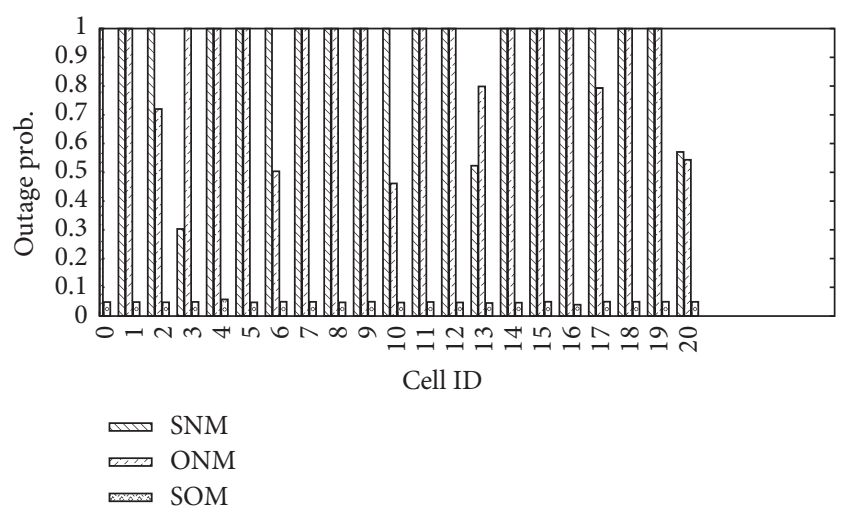

FIGURE 5: Maximum outage probability provided to a MS associated with a cell.

\section{Conclusions}

In this paper, we propose a cell-guided association method. Unlike other cell load aware methods where MSs estimate the load of neighboring cells before attempting to make associations with a cell, in CGAM, a cell decides whether or not to accept an association request from a MS. Considering the amount of available resources, a cell rejects the association request if it cannot provide the minimum data rate to a MS. The rejection guides a MS to try to associate with the second best cell. Thus, even if a MS selfishly selects a cell to maximize its benefit, they are not densely populated in some cells while the other cells are sparsely populated. Therefore, CGAM increases not only the resource utilization of a system but also the quality of service perceived by MSs. In addition, CGAM does not require additional information in a cell broadcast message and conventional cell selection metric of a MS.

\section{Competing Interests}

The author declares that there is no conflict of interests regarding the publication of this paper.

\section{Acknowledgments}

This work was partly supported by the Basic Science Research Program through the National Research Foundation of Korea (NRF) funded by the Ministry of Education (NRF-2015R1D1A1A01060117) and by the GRRC program of Gyeonggi Province [(GRRC SUWON2016-B2), Study on Object Recognition System for Industrial Security and Intelligent Control System for Establishing Social Safety Net Based on Advanced Neuro-Fuzzy Technologies].

\section{References}

[1] D. Evans, "The Internet of Things: How the Next Evolution of the Internet is Changing Everything," white paper, April 2011.

[2] Cisco, "Cisco Visual Networking Index: Global Mobile Data Traffic Forecast Update 2014-2019," White Paper, February 2015.

[3] J. G. Andrews, S. Singh, Q. Ye, X. Lin, and H. S. Dhillon, "An overview of load balancing in HetNets: old myths and open problems," IEEE Wireless Communications, vol. 21, no. 2, pp. 18 25, 2014.

[4] A. J. Fehske, H. Klessig, J. Voigt, and G. P. Fettweis, "Concurrent load-aware adjustment of user association and antenna tilts in self-organizing radio networks," IEEE Transactions on Vehicular Technology, vol. 62, no. 5, pp. 1974-1988, 2013.

[5] K. C. Garikipati and K. G. Shin, "Distributed association control in shared wireless networks," in Proceedings of the 10th Annual IEEE Communications Society Conference on Sensing and Communication in Wireless Networks (SECON '13), pp. 362370, New Orleans, La, USA, June 2013.

[6] C. Bottai, C. Cicconetti, A. Morelli, M. Rosellini, and C. Vitale, "Energy-efficient user association in extremely dense small cell networks," in Proceedings of the European Conference on Networks and Communications (EuCNC '14), pp. 1-5, Bologna, Italy, June 2014.

[7] L. P. Qian, Y. J. A. Zhang, Y. Wu, and J. Chen, "Joint base station association and power control via Benders' decomposition," IEEE Transactions on Wireless Communications, vol. 12, no. 4, pp. 1651-1665, 2013.

[8] S. Corroy, L. Falconetti, and R. Mathar, "Cell association in small heterogeneous networks: downlink sum rate and min rate maximization," in Proceedings of the IEEE Wireless Communications and Networking Conference (WCNC '12), pp. 888-892, Paris, France, April 2012.

[9] K. Shen and W. Yu, "Distributed pricing-based user association for downlink heterogeneous cellular networks," IEEE Journal on 
Selected Areas in Communications, vol. 32, no. 6, pp. 1100-1113, 2014.

[10] Q. Ye, B. Rong, Y. Chen, M. Al-Shalash, C. Caramanis, and J. G. Andrews, "User association for load balancing in heterogeneous cellular networks," IEEE Transactions on Wireless Communications, vol. 12, no. 6, pp. 2706-2716, 2013.

[11] H. Boostanimehr and V. K. Bhargava, "Unified and distributed QoS-driven cell association algorithms in heterogeneous networks," IEEE Transactions on Wireless Communications, vol. 14, no. 3, pp. 1650-1662, 2015.

[12] D. Kumar, E. Altman, and J.-M. Keli, "Globally optimal userNetwork Association in an 802.11 WLAN \& 3G UMTS hybrid cell," in Proceedings of the 20th International Teletraffic Conference on Managing Traffic Performance in Converged Networks, pp. 1173-1187, Ottawa, Canada, June 2007.

[13] C. Sun, E. Stevens-Navarro, and V. W. S. Wong, "A constrained MDP-based vertical handoff decision algorithm for $4 \mathrm{G}$ wireless networks," in Proceedings of the IEEE International Conference on Communications (ICC '08), pp. 2169-2174, IEEE, Beijing, China, May 2008.

[14] K. Son, S. Chong, and G. De Veciana, "Dynamic association for load balancing and interference avoidance in multi-cell networks," IEEE Transactions on Wireless Communications, vol. 8, no. 7, pp. 3566-3576, 2009.

[15] K. Chitti, Q. Kuang, and J. Speidel, "Joint base station association and power allocation for uplink sum-rate maximization," in Proceedings of the IEEE 14th Workshop on Signal Processing Advances in Wireless Communications (SPAWC '13), pp. 6-10, Darmstadt, Germany, June 2013.

[16] P. Wang, W. Song, D. Niyato, and Y. Xiao, "QoS-aware cell association in 5G heterogeneous networks with massive MIMO," IEEE Network, vol. 29, no. 6, pp. 76-82, 2015.

[17] L. Jiang, S. Parekh, and J. Walrand, "Base station association game in multi-cell wireless networks," in Proceedings of the IEEE Wireless Communications and Networking Conference (WCNC '08), pp. 1616-1621, Las Vegas, Nev, USA, March 2008.

[18] Y. Chen, J. Li, Z. Lin, G. Mao, and B. Vucetic, "User association with unequal user priorities in heterogeneous cellular networks," IEEE Transactions on Vehicular Technology, vol. 65, no. 9, pp. 7374-7388, 2016.

[19] S. Chen, M. Peng, H. Zhang, and C. Wang, "Investigation of service success probability for downlink heterogeneous cellular networks with cell association and user scheduling," in Proceedings of the IEEE Wireless Communications and Networking Conference (WCNC '15), pp. 1434-1439, IEEE, New Orleans, La, USA, March 2015.

[20] B. Rengarajan and G. De Veciana, "Practical adaptive user association policies for wireless systems with dynamic interference," IEEE/ACM Transactions on Networking, vol. 19, no. 6, pp. 16901703, 2011.

[21] W. Tang, S. Feng, Y. Liu, and M. C. Reed, "Joint low-power transmit and cell association in heterogeneous networks," in Proceedings of the 58th IEEE Global Communications Conference (GLOBECOM '15), pp. 1-6, IEEE, San Diego, Calif, USA, December 2015.

[22] Y. Zhu, Z. Zeng, T. Zhang, L. An, and L. Xiao, "An energy efficient user association scheme based on cell sleeping in LTE heterogeneous networks," in Proceedings of the IEEE International Symposium on Wireless Personal Multimedia Communications (WPMC '14), pp. 75-79, September 2014.

[23] H. Pervaiz, L. Musavian, and Q. Ni, "Joint user association and energy-efficient resource allocation with minimum-rate constraints in two-tier HetNets," in Proceedings of the IEEE 24th Annual International Symposium on Personal, Indoor, and Mobile Radio Communications (PIMRC '13), pp. 1634-1639, London, UK, September 2013.

[24] V. N. Ha and L. B. Le, "Distributed base station association and power control for heterogeneous cellular networks," IEEE Transactions on Vehicular Technology, vol. 63, no. 1, pp. 282-296, 2014.

[25] A. M. Rao, A. Weber, S. Gollamudi, and R. Soni, "LTE and HSPA+: revolutionary and evolutionary solutions for global mobile broadband," Bell Labs Technical Journal, vol. 13, no. 4, pp. 7-34, 2009.

[26] S. S. Mwanje and A. Mitschele-Thiel, "Minimizing handover performance degradation due to LTE self organized mobility load balancing," in Proceedings of the IEEE 77th Vehicular Technology Conference (VTC Spring '13), June 2013.

[27] 3GPP TR 36.942 Ver. 11.0.0 Rel. 11, “Technical Specification Group Radio Access Network; Evolved Universal Terrestrial Radio Access (EUTRA); Radio Frequency (RF) system scenarios," Tech. Rep., 2012.

[28] R. Combes, Z. Altman, and E. Altman, "Scheduling gain for frequency-selective Rayleigh-fading channels with application to self-organizing packet scheduling," Performance Evaluation, vol. 68, no. 8, pp. 690-709, 2011.

[29] R. Combes, S. E. Elayoubi, and Z. Altman, "Cross-layer analysis of scheduling gains: application to LMMSE receivers in frequency-selective Rayleigh-fading channels," in Proceedings of the IEEE International Symposium on Modeling and Optimization in Mobile, Ad Hoc and Wireless Networks (WiOpt '11), pp. 133-139, Princeton, NJ, USA, May 2011.

[30] H. J. Kushner and P. A. Whiting, "Convergence of proportionalfair sharing algorithms under general conditions," IEEE Transactions on Wireless Communications, vol. 3, no. 4, pp. 1250-1259, 2004.

[31] C. Tellambura, "Computing the outage probability in mobile radio networks using the sampling theorem," IEEE Transactions on Communications, vol. 47, no. 8, pp. 1125-1128, 1999.

[32] S. Tang, B. L. Mark, and A. E. Leu, "An exact solution for outage probability in cellular networks," in Proceedings of the Military Communications Conference (MILCOM '07), October 2007.

[33] H. Boostanimehr and V. K. Bhargava, "Distributed and QoSdriven cell association in HetNets to minimize global outage probability," in Proceedings of the IEEE Global Communications Conference (GLOBECOM '14), pp. 3665-3671, IEEE, Austin, Tex, USA, December 2014. 

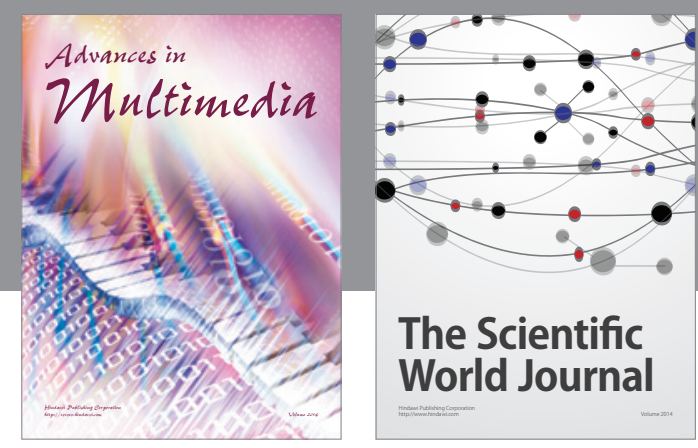

The Scientific World Journal
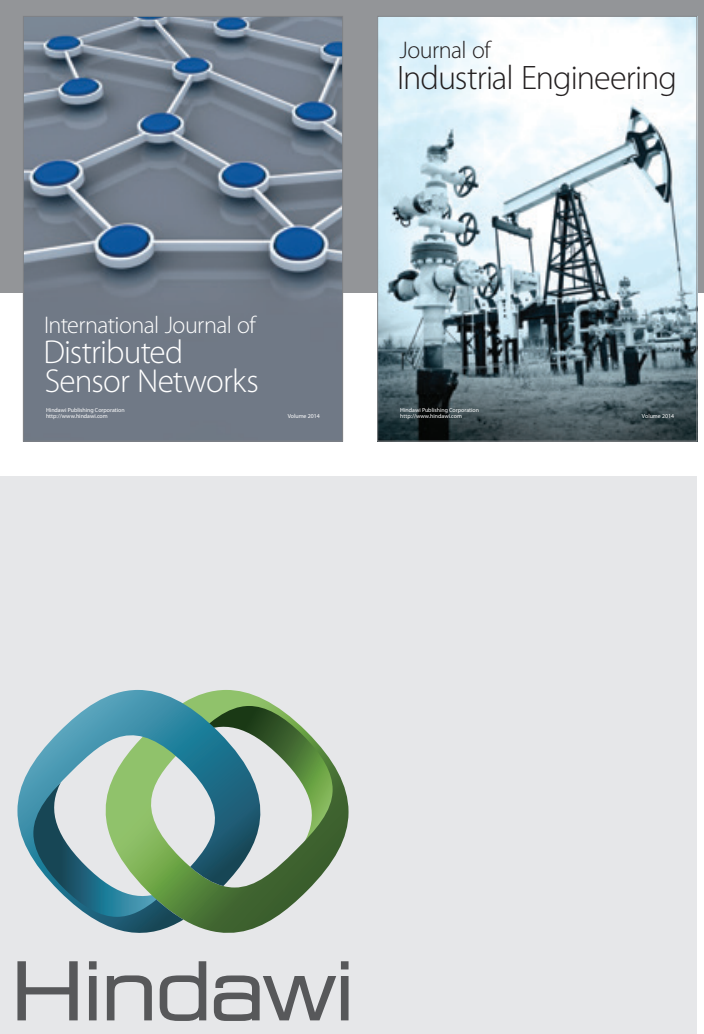

Submit your manuscripts at

https://www.hindawi.com

\section{Computer Networks} and Communications
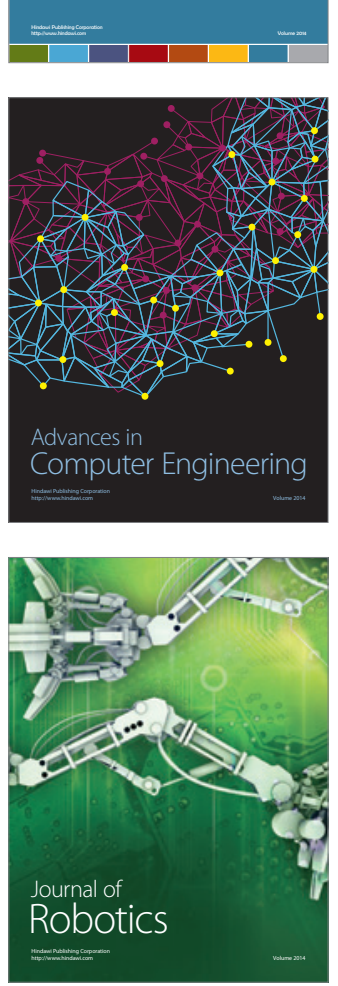
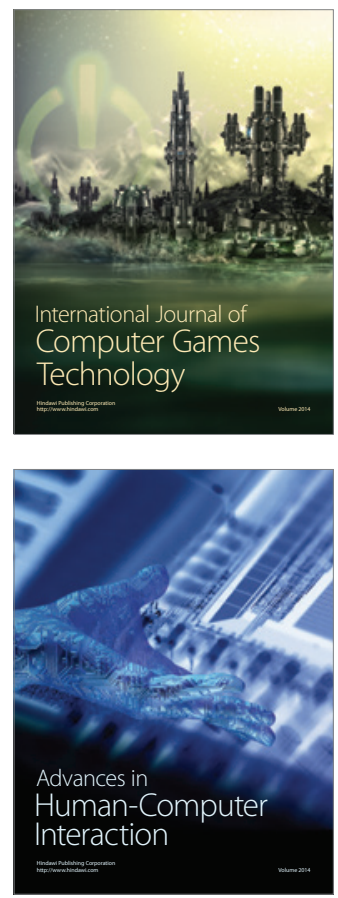
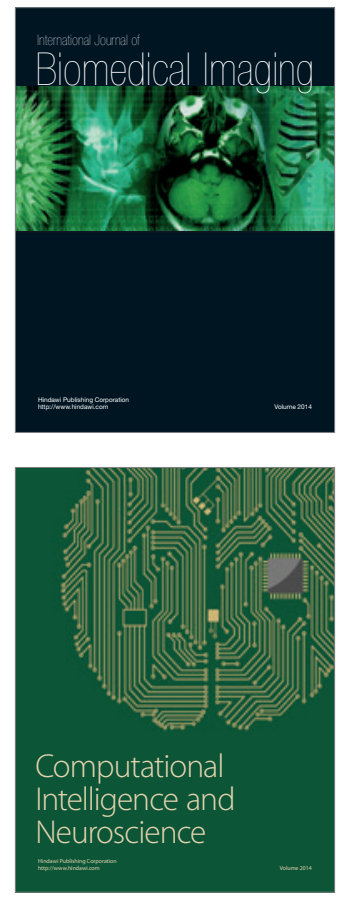
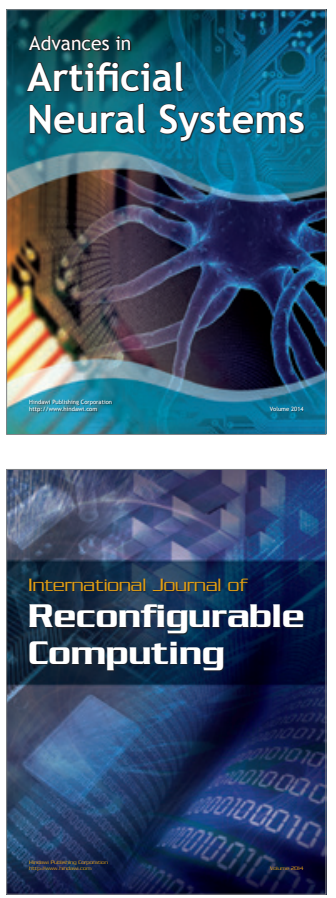
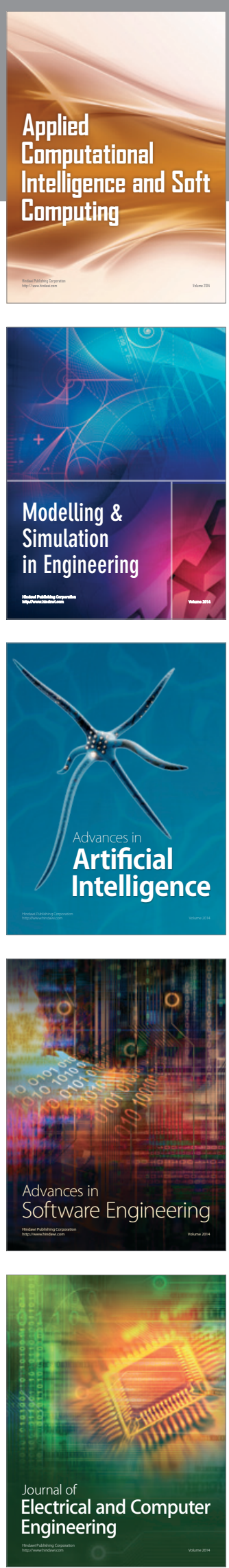